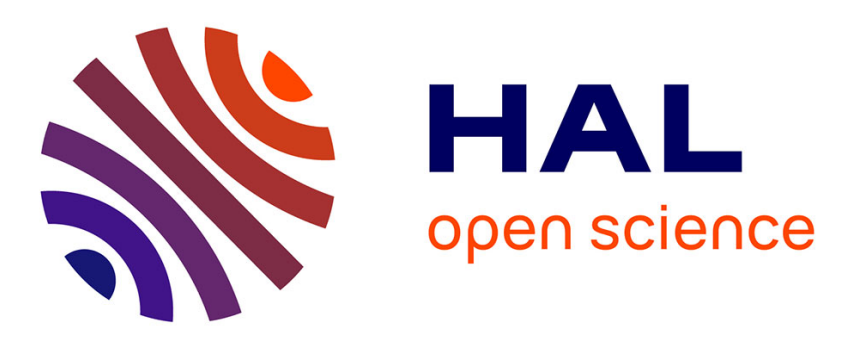

\title{
Behavior of reed woodwind instruments around the oscillation threshold
}

Benjamin Ricaud, Philippe Guillemain, Jean Kergomard, Fabrice Silva, Christophe Vergez

\section{- To cite this version:}

Benjamin Ricaud, Philippe Guillemain, Jean Kergomard, Fabrice Silva, Christophe Vergez. Behavior of reed woodwind instruments around the oscillation threshold. Acta Acustica united with Acustica, 2009, 95 (4), pp.733-743. 10.3813/AAA.918201 . hal-00355058v2

\section{HAL Id: hal-00355058 https://hal.science/hal-00355058v2}

Submitted on 23 Jan 2009

HAL is a multi-disciplinary open access archive for the deposit and dissemination of scientific research documents, whether they are published or not. The documents may come from teaching and research institutions in France or abroad, or from public or private research centers.
L'archive ouverte pluridisciplinaire HAL, est destinée au dépôt et à la diffusion de documents scientifiques de niveau recherche, publiés ou non, émanant des établissements d'enseignement et de recherche français ou étrangers, des laboratoires publics ou privés. 


\title{
Behavior of reed woodwind instruments around the oscillation threshold
}

\author{
B. Ricaud, P. Guillemain, J. Kergomard, F. Silva, C. Vergez \\ Laboratoire de Mécanique et d'Acoustique - CNRS UPR 7051 \\ 31 Ch. Joseph Aiguier, 13402 Marseille cedex 20, France. \\ Email: ricaud@lma.cnrs-mrs.fr
}

January 23, 2009

\begin{abstract}
Properties of small amplitude oscillations of the single reed woodwind instruments near the oscillation threshold are investigated. Analytical formulae with explicit dependence on the physical parameters of the instrument and instrumentist allow to determine the bifurcation point, the nature of the bifurcation, the amplitude of the first harmonics and the oscillation frequency. The model, which takes the reed dynamics into account, is entirely expressed in the frequency domain and its behaviour is analysed. An application to a model of saxophone is proposed and compared with numerical results obtained through the harmonic balance technique and a time domain simulation, showing excellent agreements.
\end{abstract}

\section{Introduction}

Woodwind musical instruments are nonlinear dynamical systems and, as such, address numerous important physical questions. The blowing pressure initiated by the instrumentalist controls the existence and the amplitude of the oscillating regime, giving the musical sound. A great challenge is to relate the physical parameters of the instrument and the player to the characteristics of the sound. Several authors have already investigated the spectrum of musical reed instruments in the steady state oscillating regime, Refs. [1, 2, 3] for a first approach using the harmonic balance technique (HBT), and some others have

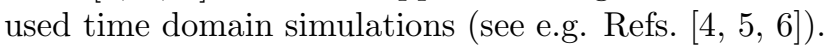

The present work is related to the HBT in the limit of small oscillations. A complete numerical version of the HBT can be found in Refs. [3, 月, 8], but previously, for small oscillations, Worman [1] suggested that a relationship exists between the amplitude of the higher harmonics, and that of the first one. Grand et al. [9], using the model of a reed without dynamics, got important analytical results, confirming the validity of the so-called Worman rule when the equations of the HBT are truncated to a finite number of harmonics. They exhibited interesting properties of the pressure spectrum inside the instrument: in the vicinity of the oscillation threshold, a simplication of the system can be done, making possible the expression of short and precise mathematical equations relating parameters with the spectrum.

Moreover, a part of the behaviour at large oscillation amplitude depends on characteristics of the small amplitude regime. Indeed, the analysis of this regime provides solutions of the system that the instrument can follow (sometimes furtively) during the play. This is why this bifurcation area is so important. Nevertheless, it must be noted that not all the possible solutions are found by the analysis of small oscillations.

Other approximate methods related to the HBT have also been studied: to obtain information around the bifurcation, the describing function (or first harmonic) method [10] is the simplest one. This consists in setting to zero all the harmonics of a periodic signal except the first one (at the fundamental frequency) and injecting it into the system of equations. Although powerful, this technique gives only limited results about the system. In Ref. [11, an approximate method, generalizing the first harmonic method, is used in order to get analytical results at small and large amplitudes for clarinet-like instruments (this study is restricted to the case of a quasi-cylindrical resonator and considers the reed as a simple spring). Another kind of approximation is also used in Ref. [12] in order to treat conical reed instruments. 
In the previously cited works, attempts to treat the effect of reed dynamics can be found in [1, 9]. A more complete treatment has been published in Ref. [13].

The goal of the present paper is first to present an even more general model, taking into account a reed dynamics which needs not to be explicit, and to settle the full harmonic balance equations. Then it aims to obtain analytical results from these equations, with the help of simplifications allowed by the so called Worman rule for small oscillations, extending the results of Ref. [9]. In this study, the reed is supposed to be non-beating and the pressure difference between mouth and reed is assumed to be positive (this implicitly assumes an oscillating regime of small to moderate amplitude, with positive acoustic flow). These two assumptions lead to a simple polynomial expression for the nonlinear relation of the system, and allow to obtain the full harmonic balance equations in the frequency domain (Sec. 2). Then, in Sec. 3, the assumption of a small oscillation is made, implying the Worman rule [1, 9]. As a consequence, it allows simplifications of the equations, since the high frequency harmonics can be neglected. In the following sections, it is shown that already known results for several particular cases are retrieved by this more general approach. New relations are presented concerning the nature of the bifurcation and the frequency dependence with respect to the blowing pressure (Sec. A). In the particular case of reed modeled as a simple spring, links are made with previous studies (Sec. 5). Eventually, the results are compared with a numerical harmonic balance method and a time domain simulation (Sec. 6).

\section{The model}

The model of reed woodwind instruments studied here is detailed in $[13$ and in references therein and will not be discussed here. In conformity with these studies, all quantities introduced in the following are dimensionless. Let us call $p(t)$ and $u(t)$ the acoustic pressure and volume flow, respectively, in the mouthpiece entry at time $t$ and $x(t)$ the reed tip opening. Considering the steady-state oscillating regime, all these functions are assumed to be periodic. The Fourier transforms of these quantities will be denoted with a capital letter or a hat. More precisely, for a periodic pressure signal of period $\tau$, the $n$th harmonic of $p$ is:

$$
P\left(\omega_{n}\right)=P_{n}=\widehat{p}\left(\omega_{n}\right)=\frac{1}{\tau} \int_{0}^{\tau} p(t) e^{-i \omega_{n} t} d t, \quad \omega_{n}=\frac{2 \pi n}{\tau} .
$$

The input impedance $Z$ of the tube links the pressure and the volume flow in the frequency domain through:

$$
P(\omega)=Z(\omega) U(\omega)
$$

It takes into account the resonator acoustical behavior and, possibly, the reed induced flow. A detailed study of this impedance can be found e.g. in Ref. [14. Like in Ref. [13], it is assumed here that $Z(0)=0$ (this assumption allows a great simplification of the equations, but does not change the basic principle of the small oscillations calculation, as shown in Ref. [9]). It is also assumed that for all $\omega \neq 0, Z(\omega) \neq 0$, thus it can be written:

$$
U(\omega)=Y(\omega) P(\omega), \quad \text { for all } \omega \neq 0,
$$

where $Y$ is the input admittance of the tube, which can take into account the reed induced flow. The reed aperture is related to the pressure in the mouthpiece by the following transfer function $D$ :

$$
X(\omega)=D(\omega) P(\omega) .
$$

When considering only the first flexural vibration mode of the reed, $D(\omega)$ may reduce to a second-order lowpass resonant filter. Eventually, for small oscillations, which implies a non-beating reed, there is a nonlinear relationship at the tip of the mouthpiece. Using some hypotheses, it is given by the Bernoulli relationship in the time domain and the assumption of a rectangular reed channel:

$$
u^{2}(t)=\zeta^{2}(1-\gamma+x(t))^{2}(\gamma-p(t))
$$

where $\zeta$ is related to the reed and player parameters as defined in Ref. [13] and $\gamma$ is related to the pressure in the mouth of the musician. This latter is chosen to be the bifurcation parameter. It is supposed that $\gamma>p(t)$ : this is true at least for small oscillations as the value of $\gamma$ at threshold is strictly positive (as it will be shown in the following). It is to be noted that two solutions of Eq. (5) of opposite sign exist for $u(t)$, and the condition $u(t)>0$ is assumed to be true, as $\gamma>p(t)$. 
Let us express Eq. (5) in the frequency domain. Let us remind that for two $\tau$-periodic functions of time $f$ and $g$, the multiplication becomes in the Fourier space a convolution:

$$
\widehat{f g}\left(\omega_{q}\right)=(F * G)\left(\omega_{q}\right)=\sum_{n \in \mathbb{Z}} F\left(\omega_{q}-\omega_{n}\right) G\left(\omega_{n}\right),
$$

where $\omega_{q}=\frac{2 \pi}{\tau} q$ and $q$ is an integer. As introduced in expression (1) $F\left(\omega_{q}\right)$ may also be denoted $F_{q}$. Therefore, expressions such as the following ones are obtained:

$$
\begin{aligned}
& \widehat{u^{2}}\left(\omega_{q}\right)=(U * U)\left(\omega_{q}\right)=\sum_{n \in \mathbb{Z}} U_{q-n} U_{n}, \\
& \widehat{x p}\left(\omega_{q}\right)=(X * P)\left(\omega_{q}\right)=((D P) * P)\left(\omega_{q}\right)=\sum_{n \in \mathbb{Z}} D_{q-n} P_{q-n} P_{n} .
\end{aligned}
$$

Remark that this calculation is more straightforward than in Ref. [11]. Indeed, it uses the convolution formula instead of multiplication of Fourier series, and does not use any approximation. If the following notations are introduced:

$$
\begin{aligned}
& u_{00}=\zeta^{2} \gamma(1-\gamma)^{2} \\
& A_{q}=2 \zeta^{2} \gamma(1-\gamma) D_{q}-\zeta^{2}(1-\gamma)^{2} \\
& B_{q, n}=\zeta^{2} \gamma D_{q-n} D_{n}-2 \zeta^{2}(1-\gamma) D_{q-n} \\
& C_{q, n, m}=-\zeta^{2} D_{q-n} D_{n-m}
\end{aligned}
$$

Eq. (5) becomes in the Fourier space, for all $q$ :

$$
\sum_{n \in \mathbb{Z}} U_{q-n} U_{n}=u_{00} \delta_{q}+A_{q} P_{q}+\sum_{n \in \mathbb{Z}} B_{q, n} P_{q-n} P_{n}+\sum_{n \in \mathbb{Z}} \sum_{m \in \mathbb{Z}} C_{q, n, m} P_{q-n} P_{n-m} P_{m},
$$

where $\delta$ is the Kronecker symbol. The quantity $Y_{0}$ being infinite and $P_{0}$ being zero, the following notation is introduced:

$$
\mathbf{H}_{q, n}=B_{q, n}-Y_{q-n} Y_{n}, \quad \text { for } \quad n \neq 0, n \neq q,
$$

with the convention $\mathbf{H}_{q, 0}=B_{q, 0}, \mathbf{H}_{q, q}=B_{q, q}$ for all $q \in \mathbb{Z}$. Using equations (3), (价, and since $P_{0}=0$, Eq. (7) is written as:

$$
\begin{aligned}
\left(2 U_{0} Y_{q}-A_{q}\right) P_{q}=\sum_{n \in \mathbb{Z}} \mathbf{H}_{q, n} P_{q-n} P_{n}+\sum_{n \in \mathbb{Z}} \sum_{m \in \mathbb{Z}} C_{q, n, m} P_{q-n} P_{n-m} P_{m}, & \text { for } q \neq 0 \\
\left|U_{0}\right|^{2}=u_{00}+\sum_{n \in \mathbb{Z}} \mathbf{H}_{0, n}\left|P_{n}\right|^{2}+\sum_{n \in \mathbb{Z}} \sum_{m \in \mathbb{Z}} C_{0, n, m} P_{-n} P_{n-m} P_{m}, & \text { for } q=0 .
\end{aligned}
$$

These equations are the complete harmonic balance equations.

Let us make some remarks:

i) it can be noticed that the static solution $U_{0}=\sqrt{u_{00}}$, where for all $n \in \mathbb{Z}, P_{n}=0$, remains valid for all $\gamma$.

ii) In this study, only the case $a<\gamma<b$ will be studied where $a, b$ are such that $0<a<b<1$. In fact these two constants are chosen in order to satisfy $1-\gamma+x(t)>0$ and $\gamma-p(t)>0$. This is a consequence of the hypothesis that $u(t)$ is positive at small oscillations and of Eq. (5). At small oscillations, $p(t), x(t)$ are small so $a$ is close to 0 and $b$ is close to 1 .

iii) the time domain functions $p, u$ are real so $P_{-n}=\overline{P_{n}}$, therefore only the case $n \geq 0$ needs to be treated. The quantity $P_{1}$ can be chosen to be real but nothing requires this in the study so it is supposed to be a complex number in the following. 


\section{The bifurcation threshold}

Near the oscillation threshold, where $P_{1}$ is small, we apply the so called "Worman rule" (see e.g. [1, 91), and the amplitude of each harmonic of the pressure is proportional to a power of $P_{1}$ :

$$
P_{q}=\mathcal{O}\left(\left|P_{1}\right|^{|q|}\right), \quad P_{0}=0 .
$$

As a consequence, for small oscillations the higher harmonics are small and Eq. (9a) can be reduced, after a little calculation, to:

$$
\left(2 U_{0} Y_{q}-A_{q}\right) P_{q}=\sum_{n=1}^{q-1} \mathbf{H}_{q, n} P_{q-n} P_{n}+\sum_{n=1}^{q-1} \sum_{m=1}^{n-1} C_{q, n, m} P_{q-n} P_{n-m} P_{m}+\mathcal{O}\left(\left|P_{1}\right|^{q+1}\right), \quad \text { for } q>1 .
$$

Each term of the sums is of order $\mathcal{O}\left(\left|P_{1}\right|^{q}\right)$, in agreement with the Worman rule. For the case $q=1$ Eq. (9a) gives

$$
\left(2 U_{0} Y_{1}-A_{1}\right) P_{1}=\left(\mathbf{H}_{1,-1}+\mathbf{H}_{1,2}\right) P_{-1} P_{2}+\left(C_{1,0,1}+C_{1,0,-1}+C_{1,2,1}\right)\left|P_{1}\right|^{2} P_{1}+\mathcal{O}\left(\left|P_{1}\right|^{4}\right) .
$$

Keeping the terms up to order three in $P_{1}$, Eq. (9b) reduces to

$$
\left|U_{0}\right|^{2}=u_{00}+\left(\mathbf{H}_{0,1}+\mathbf{H}_{0,-1}\right)\left|P_{1}\right|^{2}+\mathcal{O}\left(\left|P_{1}\right|^{4}\right) .
$$

Let us introduce the notation

$$
\begin{aligned}
\mathbf{K}_{n} & =2 \sqrt{u_{00}} Y_{n}-A_{n} \\
& =2 \zeta^{2} \gamma(1-\gamma)\left(\frac{Y_{n}}{\zeta \sqrt{\gamma}}+\frac{1-\gamma}{2 \gamma}-D_{n}\right) .
\end{aligned}
$$

The function $\mathbf{K}_{n}$ depends on both $\gamma$ and $\omega$ and will be written $\mathbf{K}_{n}, \mathbf{K}_{n}(\gamma), \mathbf{K}\left(\gamma, \omega_{n}\right)$ whenever emphasis is needed on these variables.

Suppose now that the system is oscillating at frequency $\omega(\gamma)$, with a small amplitude, and the parameter $\gamma$ varies such that the amplitude of oscillation tends to zero and reaches zero for some $\gamma=\gamma_{0}$. From (13) this implies $\left|U_{0}\right|^{2}=u_{00}$ and since the volume flow is assumed to be positive for small oscillation, this leads to $U_{0}=\sqrt{u_{00}}$. For $q \neq 1$ in (9), the Worman rule is satisfied and each harmonic tends to zero. But for the case $q=1$, this leads to

$$
\left(2 U_{0} Y_{1}-A_{1}\right) P_{1}=\mathcal{O}\left(P_{1}^{3}\right) .
$$

Eliminating the solution $P_{1}=0$ corresponding to the static regime, this latter formula implies that $\left(2 U_{0} Y_{1}-A_{1}\right)$ is proportional to $\left|P_{1}\right|^{2}$ and at $\gamma_{0}$

$$
2 \sqrt{u_{00}} Y_{1}-A_{1}=\mathbf{K}\left(\gamma_{0}, \omega_{1}\left(\gamma_{0}\right)\right)=0 .
$$

It is a standard property of nonlinear systems that the frequency of oscillation depends on the amplitude of oscillation, (see e.g [16]) and the above formula determines the critical parameter $\gamma_{0}$ and the angular frequency at threshold

$$
\omega_{1}^{0}=\omega_{1}\left(\gamma_{0}\right) .
$$

As $\mathbf{K}\left(\gamma_{0}, \omega_{1}^{0}\right)$ is a complex number, this leads to a set of two equations:

$$
\left\{\begin{array}{c}
\frac{\mathcal{R} e\left[Y\left(\omega_{1}^{0}\right)\right]}{\zeta \sqrt{\gamma_{0}}}+\frac{1-\gamma_{0}}{2 \gamma_{0}}-\mathcal{R} e\left[D\left(\omega_{1}^{0}\right)\right]=0, \\
\frac{\mathcal{I m}\left[Y\left(\omega_{1}^{0}\right)\right]}{\zeta \sqrt{\gamma_{0}}}-\mathcal{I} m\left[D\left(\omega_{1}^{0}\right)\right]=0 .
\end{array}\right.
$$

This result is exactly the one obtained in Ref. [13] by linearization, (see formulae (8) and (9)). Notice that in order to obtain this result, it must be shown that the harmonics with indices greater than one are negligible compared to the fundamental one, when the oscillation amplitude tends to zero. So a property less restrictive than the Worman rule could have been used. 


\section{Amplitude and frequency around $\gamma_{0}$}

In this section the oscillating regime of the system around the bifurcation point is investigated. The amplitude of oscillation will be shown to be small and here again, the rule given in Eq. (10) is used. Under this condition, a preliminary calculus is done showing an important relationship deduced from Eqs. (9). Then the direction of bifurcation is given, together with the amplitude of the first three harmonics and the variation of the frequency with respect to $\gamma$.

\subsection{Preliminaries}

When the amplitude of oscillation is small, for values of $\gamma$ near $\gamma_{0}$, Eq. (13) (with $U_{0}>0$ ) can be reduced to

$$
U_{0}=\sqrt{u_{00}}+\frac{\left(\mathbf{H}_{0,1}+\mathbf{H}_{0,-1}\right)}{2 \sqrt{u_{00}}}\left|P_{1}\right|^{2}+\mathcal{O}\left(\left|P_{1}\right|^{4}\right)
$$

Therefore, using the definition of $\mathbf{K}_{1}$ :

$$
2 U_{0} Y_{1}-A_{1}=\mathbf{K}_{1}+\frac{\left(\mathbf{H}_{0,1}+\mathbf{H}_{0,-1}\right)}{\sqrt{u_{00}}} Y_{1}\left|P_{1}\right|^{2}+\mathcal{O}\left(\left|P_{1}\right|^{4}\right) .
$$

Similarly, Eq. (11) gives for $q=2$ the following result:

$$
\left(\mathbf{K}_{2}+\mathcal{O}\left(\left|P_{1}\right|^{2}\right)\right) P_{2}=\mathbf{H}_{2,1} P_{1} P_{1}+\mathcal{O}\left(\left|P_{1}\right|^{4}\right)
$$

thus

$$
P_{2}=\frac{\mathbf{H}_{2,1}}{\mathbf{K}_{2}} P_{1}^{2}+\mathcal{O}\left(\left|P_{1}\right|^{4}\right)
$$

Together with (12), this leads to the following equation:

$$
\begin{aligned}
& \mathbf{K}_{1} P_{1}+\frac{\left(\mathbf{H}_{0,1}+\mathbf{H}_{0,-1}\right)}{\sqrt{u_{00}}} Y_{1}\left|P_{1}\right|^{2} P_{1}+\mathcal{O}\left(\left|P_{1}\right|^{5}\right)= \\
& =\left(\left(\mathbf{H}_{1,2}+\mathbf{H}_{1,-1}\right) \frac{\mathbf{H}_{2,1}}{\mathbf{K}_{2}}+C_{1,0,1}+C_{1,0,-1}+C_{1,2,1}\right)\left|P_{1}\right|^{2} P_{1}+\mathcal{O}\left(\left|P_{1}\right|^{5}\right)
\end{aligned}
$$

Let us introduce the notation:

$$
Q_{1}(\gamma)=\left(\mathbf{H}_{1,2}+\mathbf{H}_{1,-1}\right) \frac{\mathbf{H}_{2,1}}{\mathbf{K}_{2}}+C_{1,0,1}+C_{1,0,-1}+C_{1,2,1}-\frac{\left(\mathbf{H}_{0,1}+\mathbf{H}_{0,-1}\right)}{\sqrt{u_{00}}} Y_{1} .
$$

The reader must keep in mind that $Q_{1}$ depends on $\gamma, \omega_{1}, Y_{1}, Y_{2}, D_{1}, D_{2}$ and $\zeta$. Then, for small oscillation

$$
\mathbf{K}_{1}(\gamma) P_{1}-Q_{1}(\gamma)\left|P_{1}\right|^{2} P_{1}=\mathcal{O}\left(\left|P_{1}\right|^{5}\right)
$$

which implies for the case $P_{1} \neq 0$ :

$$
\left|P_{1}\right|^{2}=\frac{\mathbf{K}_{1}(\gamma)}{Q_{1}(\gamma)}+\mathcal{O}\left(\left|P_{1}\right|^{4}\right)
$$

This is exactly the result of 13] (see Eq.(50)). Here an indication of the error term is given. Calculating the Taylor expansion around $\gamma_{0}$ and since $\mathbf{K}_{1}\left(\gamma_{0}\right)=0$, the following result is obtained:

$$
\left|P_{1}\right|^{2}=\left\{\mathcal{R} e\left[\frac{\mathbf{K}_{1}^{\prime}\left(\gamma_{0}\right)}{Q_{1}\left(\gamma_{0}\right)}\right]+i \mathcal{I} m\left[\frac{\mathbf{K}_{1}^{\prime}\left(\gamma_{0}\right)}{Q_{1}\left(\gamma_{0}\right)}\right]\right\} \cdot\left(\gamma-\gamma_{0}\right)+\mathcal{O}\left(\gamma-\gamma_{0}\right)^{2}+\mathcal{O}\left(\left|P_{1}\right|^{4}\right)
$$

where $\mathbf{K}_{1}^{\prime}\left(\gamma_{0}\right)$ is the total derivative of $\mathbf{K}_{1}$ with respect to $\gamma$ at $\gamma_{0}$, its expression being:

$$
\mathbf{K}_{1}^{\prime}\left(\gamma_{0}\right)=\frac{d \omega}{d \gamma}\left(\gamma_{0}\right) \cdot \frac{\partial \mathbf{K}_{1}}{\partial \omega}\left(\omega\left(\gamma_{0}\right), \gamma_{0}\right)+\frac{\partial \mathbf{K}_{1}}{\partial \gamma}\left(\omega\left(\gamma_{0}\right), \gamma_{0}\right)
$$


Eq. (22) immediately shows that $\left|P_{1}\right|$ is proportional to $\sqrt{\left|\gamma-\gamma_{0}\right|}$ for $\gamma$ close to $\gamma_{0}$ and the estimation $\mathcal{O}\left(\left|P_{1}\right|^{4}\right)$ can be replaced by $\mathcal{O}\left(\left|\gamma-\gamma_{0}\right|^{2}\right)$ in the following. Secondly, as the left hand side is a real, positive quantity, Eq. (22) implies

$$
\mathcal{I} m\left[\frac{\mathbf{K}_{1}^{\prime}\left(\gamma_{0}\right)}{Q_{1}\left(\gamma_{0}\right)}\right]=\frac{\mathcal{I} m \mathbf{K}_{1}^{\prime}\left(\gamma_{0}\right) \mathcal{R} e Q_{1}\left(\gamma_{0}\right)-\mathcal{R} e \mathbf{K}_{1}^{\prime}\left(\gamma_{0}\right) \mathcal{I} m Q_{1}\left(\gamma_{0}\right)}{\left|Q_{1}\left(\gamma_{0}\right)\right|^{2}}=0
$$

or

$$
\mathcal{I} m \mathbf{K}_{1}^{\prime}\left(\gamma_{0}\right)=\mathcal{R} e \mathbf{K}_{1}^{\prime}\left(\gamma_{0}\right) \frac{\mathcal{I} m Q_{1}\left(\gamma_{0}\right)}{\mathcal{R} e Q_{1}\left(\gamma_{0}\right)}
$$

In the next section, it will be shown that this relationship determines the slope of the oscillation frequency when $\gamma$ varies. Using the notation

$$
\theta=\frac{\mathcal{I} m Q_{1}\left(\gamma_{0}\right)}{\mathcal{R} e Q_{1}\left(\gamma_{0}\right)}=\frac{\mathcal{I} m \mathbf{K}_{1}^{\prime}\left(\gamma_{0}\right)}{\mathcal{R} e \mathbf{K}_{1}^{\prime}\left(\gamma_{0}\right)}
$$

the quantity $\theta$ depends on $\gamma_{0}, \omega_{1}^{0}=\omega_{1}\left(\gamma_{0}\right), \zeta, Y_{1}, D_{1}$ the transfer functions taken at $\omega_{1}^{0}$ and $Y_{2}, D_{2}$ taken at $2 \omega_{1}^{0}$. Therefore,

$$
\mathcal{R} e\left[\frac{\mathbf{K}_{1}^{\prime}\left(\gamma_{0}\right)}{Q_{1}\left(\gamma_{0}\right)}\right]=\frac{\mathcal{R} e\left[\mathbf{K}_{1}^{\prime}\left(\gamma_{0}\right)\right](1+i \theta)}{\mathcal{R} e\left[Q_{1}\left(\gamma_{0}\right)\right](1+i \theta)}=\frac{\mathcal{R} e\left[\mathbf{K}_{1}^{\prime}\left(\gamma_{0}\right)\right]}{\mathcal{R} e\left[Q_{1}\left(\gamma_{0}\right)\right]}
$$

Let us introduce:

$$
\alpha=\frac{\mathcal{R} e\left[\mathbf{K}_{1}^{\prime}\left(\gamma_{0}\right)\right]}{\mathcal{R} e\left[Q_{1}\left(\gamma_{0}\right)\right]} .
$$

This quantity depends on $D, Y$ and the derivative of $Y$ with respect to $\omega$ at frequency $\omega_{1}^{0}$, as well as $D$ at $2 \omega_{1}^{0}, \gamma$ and $\zeta$, as can be seen in appendix A. Finally :

$$
\left|P_{1}\right|^{2}=\alpha \cdot\left(\gamma-\gamma_{0}\right)+\mathcal{O}\left(\gamma-\gamma_{0}\right)^{2}
$$

\subsection{Direction of the bifurcation}

Since the left hand side of $(28)$ is positive, the direction of bifurcation (oscillations for $\gamma<\gamma_{0}$ or $\gamma>\gamma_{0}$ ) is given by the sign of $\mathcal{R} e \mathbf{K}_{1}^{\prime}\left(\gamma_{0}\right) \mathcal{R} e Q_{1}\left(\gamma_{0}\right)$. As a consequence, distinguishing the two cases, the bifurcation is direct or inverse, as follows :

\begin{tabular}{|c|c|}
\hline For $\mathcal{R} e \mathbf{K}_{1}^{\prime}\left(\gamma_{0}\right) \mathcal{R} e Q_{1}\left(\gamma_{0}\right)>0$ & Direct bifurcation, small oscillation exists for $\gamma>\gamma_{0}$ \\
\hline For $\mathcal{R} e \mathbf{K}_{1}^{\prime}\left(\gamma_{0}\right) \mathcal{R} e Q_{1}\left(\gamma_{0}\right)<0$ & Inverse bifurcation, small oscillation exists for $\gamma<\gamma_{0}$ \\
\hline
\end{tabular}

The general properties of a static-oscillating regime transition (Hopf bifurcation) state that the oscillating regime is stable for a direct bifurcation and unstable for an inverse one, see e.g. [15, sec. 9.3] or [16, sec. 1.4] (Notice that a direct bifurcation is also called supercritical and the inverse one, subcritical). The sign of $\mathcal{R} e \mathbf{K}_{1}^{\prime}\left(\gamma_{0}\right) \mathcal{R} e Q_{1}\left(\gamma_{0}\right)$ is then a simple criterion to determine whether small oscillations (i.e. pianissimo notes) can be produced in practice in a reed instrument. The quantity $\mathbf{K}_{1}^{\prime}\left(\gamma_{0}\right)$ only depends on the values of the transfer functions $Y$ and $D$ and their respective derivative at frequency $\omega_{1}$ from Eq. (18), while $Q_{1}$ depends only on $Y$ and $D$ at frequencies $\omega_{1}$ and $2 \omega_{1}$ (from Eq. (20)). It is remarkable that the direction of bifurcation does not depend on values of theses quantities at higher frequencies, as noted in [9], but this can be understood with this analytic harmonic balance approach: even the roughest estimation of $P_{1}$ given by (28) must satisfy this condition of existence. Notice that $\gamma_{0}$ is determined by the values of $Y_{1}$ and $D_{1}$ only.

\subsection{Amplitude}

The analytical expression of the first three harmonics of the pressure, close to the oscillation threshold, can be deduced from the previous results. Using Eq. (28), and knowing the direction of bifurcation from the previous section, the amplitude of the first harmonic is given by:

$$
\left|P_{1}\right|=\sqrt{|\alpha|} \cdot \sqrt{\left|\gamma-\gamma_{0}\right|}+\mathcal{O}\left(\left|\gamma-\gamma_{0}\right|\right)
$$


where the quantity $\alpha$ is defined in (27). For the second harmonic, Eq. (19) gives:

$$
\left|P_{2}\right|=\left|\frac{\mathbf{H}_{2,1}}{\mathbf{K}_{2}(\gamma)}\right|_{\gamma=\gamma_{0}} \alpha \cdot\left(\gamma-\gamma_{0}\right)+\mathcal{O}\left(\gamma-\gamma_{0}\right)^{2} .
$$

Finally, for the third harmonic, from Eq. (11):

$$
\mathbf{K}_{3}(\gamma) P_{3}=\left(\mathbf{H}_{3,1}+\mathbf{H}_{3,2}\right) P_{2} P_{1}+C_{3,2,1} P_{1} P_{1} P_{1}+\mathcal{O}\left(\left|P_{1}\right|^{5}\right),
$$

and

$$
\left|P_{3}\right|=\left|\frac{\mathbf{H}_{3,1}+\mathbf{H}_{3,2}}{\mathbf{K}_{3}(\gamma)} \frac{\mathbf{H}_{2,1}}{\mathbf{K}_{2}(\gamma)}+C_{3,2,1}\right|_{\gamma=\gamma_{0}} \alpha^{\frac{3}{2}} \cdot\left(\left|\gamma-\gamma_{0}\right|\right)^{\frac{3}{2}}+\mathcal{O}\left(\left|\gamma-\gamma_{0}\right|\right)^{\frac{5}{2}} .
$$

These formulae differ from those of Ref. [11] in two ways : on the one hand they are limited to small oscillations, but on the other hand they include the effects of reed dynamics (moreover, no hypothesis is made concerning the amplitude of the even harmonics).

\subsection{Frequency shift}

Expression (24) will determine the value of the oscillation frequency around $\gamma_{0}$. For a nonlinear oscillating system, the angular frequency of the oscillation depends on the parameter $\gamma$ (see e.g. Ref. [16]). Calculating the Taylor expansion of $\omega$ around $\gamma_{0}$ gives:

$$
\omega(\gamma)=\omega_{1}^{0}+\frac{d \omega}{d \gamma}\left(\gamma_{0}\right) \cdot\left(\gamma-\gamma_{0}\right)+\mathcal{O}\left(\gamma-\gamma_{0}\right)^{2},
$$

where $\omega_{1}^{0}$ is the angular frequency at threshold obtained by solving Eq. (18). Putting Eq. (23) in Eq. (24) leads to the sought formula:

$$
\frac{d \omega}{d \gamma}\left(\gamma_{0}\right)=-\frac{\theta \frac{\partial \mathcal{R} e \mathbf{K}_{1}}{\partial \gamma}\left(\omega\left(\gamma_{0}\right), \gamma_{0}\right)-\frac{\partial \mathcal{I} m \mathbf{K}_{1}}{\partial \gamma}\left(\omega\left(\gamma_{0}\right), \gamma_{0}\right)}{\theta \frac{\partial \mathcal{R} e \mathbf{K}_{1}}{\partial \omega}\left(\omega\left(\gamma_{0}\right), \gamma_{0}\right)-\frac{\partial \mathcal{I} m \mathbf{K}_{1}}{\partial \omega}\left(\omega\left(\gamma_{0}\right), \gamma_{0}\right)} .
$$

Let us introduce the notation

$$
Y_{1}^{\prime}(\omega)=\frac{d Y_{1}}{d \omega}(\omega), \quad D_{1}^{\prime}(\omega)=\frac{d D_{1}}{d \omega}(\omega)
$$

For reed instruments, with $\mathbf{K}_{1}$ defined in Eq. (14) and since $\mathbf{K}_{1}\left(\gamma_{0}\right)=0$, this implies

$$
\begin{aligned}
& \frac{\partial \mathbf{K}_{1}}{\partial \gamma}\left(\omega\left(\gamma_{0}\right), \gamma_{0}\right)=\zeta^{2}\left(1-\gamma_{0}\right)\left(\frac{Y_{1}(\omega)}{\zeta \sqrt{\gamma_{0}}}-1-2 D_{1}(\omega)\right), \\
& \frac{\partial \mathbf{K}_{1}}{\partial \omega}\left(\omega\left(\gamma_{0}\right), \gamma_{0}\right)=\zeta^{2}\left(1-\gamma_{0}\right)\left(\frac{2 Y_{1}^{\prime}(\omega) \sqrt{\gamma_{0}}}{\zeta}-2 \gamma_{0} D_{1}^{\prime}(\omega)\right) .
\end{aligned}
$$

The equation $\mathbf{K}_{1}\left(\gamma_{0}\right)=0$ implies $2 Y_{1} /\left(\zeta \sqrt{\gamma_{0}}\right)=1-1 / \gamma_{0}+2 D_{1}$ and

$$
\frac{\partial \mathbf{K}_{1}}{\partial \gamma}\left(\omega\left(\gamma_{0}\right), \gamma_{0}\right)=\zeta^{2}\left(1-\gamma_{0}\right)\left(-\frac{1}{2}-\frac{1}{2 \gamma_{0}}-D_{1}(\omega)\right) .
$$

An application of this formula can be made for the case of a (almost) lossless cylindrical resonator. This implies that $Y_{2}$ tends to infinity, then $Q_{1}=C$, as defined in appendix A. Using the approximation $\gamma_{0}=1 / 3$, the following result is obtained:

$$
Q_{1}=-\frac{\zeta^{2}}{2}\left[2 D_{1}+\overline{D_{1}}+3\right]
$$

Then, writing the values given in Ref. [17] (see also [15, sec. 9.5.2]) for $Y_{1}, D_{1}$ and taking into account the reed induced flow:

$$
\begin{aligned}
& Y(\omega) \simeq j\left[\frac{\ell}{c}\left[\omega-\omega_{n}\right]+\frac{\omega}{c} \Delta \ell\right] \\
& D(\omega) \simeq 1-j q_{r} \omega / \omega_{r},
\end{aligned}
$$


where $c$ is the speed of sound, $j$ the imaginary number, $\ell$ is the length of the instrument, $\Delta \ell$ is the length correction due to the reed induced flow, $\omega_{n}$ the tube eigenfrequency closest to $\omega$ (assuming $\omega_{n}$ much smaller than $\omega_{r}$ ) and $\omega_{r}, q_{r}$ are the reed resonance frequency and damping factor, respectively. This gives $\theta=\operatorname{Im}\left[D_{1}\right] / 6$ and

$$
\frac{\partial \mathbf{K}_{1}}{\partial \gamma}\left(\omega\left(\frac{1}{3}\right), \frac{1}{3}\right)=-\frac{2}{3} \zeta^{2}\left(3+i \mathcal{I} m\left[D_{1}\right]\right), \quad \frac{\partial \mathbf{K}_{1}}{\partial \omega}\left(\omega\left(\frac{1}{3}\right), \frac{1}{3}\right)=i \frac{4 \zeta}{3 \sqrt{3}}\left(\mathcal{I} m\left[Y_{1}^{\prime}\right]-\frac{\zeta \mathcal{I} m\left[D_{1}^{\prime}\right]}{\sqrt{3}}\right) .
$$

Denoting $\gamma_{01}$ the approximation of $\gamma_{0}$ to the first order and $k_{r}=\omega_{r} / c$, this leads to

$$
\omega(\gamma)=\frac{\omega_{n}(\ell-\Delta \ell)}{\ell}-\zeta q_{r} \frac{1}{\sqrt{3}} \frac{\omega_{n}}{k_{r} \ell}\left[1+\frac{3}{4}\left(\gamma-\gamma_{01}\right)\right] .
$$

This formula was given in Ref. [17] and explain why at low levels of excitation the oscillation frequency decreases when the clarinetist plays harder.

\section{Case without reed dynamics}

Many results exist for reed instruments for the small oscillation regime for the case where the reed is a simple spring. This section aims to check that the present study reduced to this particular case confirm the results of previous publications. In addition, some formulae here are given in a more precise and detailed form.

If the reed is assumed to be a simple spring without dynamics, the expression of $D(\omega)$ becomes:

$$
D(\omega)=1,
$$

for all $\omega$. From Eq. (18), the oscillation angular frequency at threshold, $\omega_{1}^{0}=\omega\left(\gamma_{0}\right)$, is directly given by $\operatorname{I} m\left[Y\left(\omega_{1}^{0}\right)\right]=\operatorname{Im}\left[Y_{1}\right]=0$. The bifurcation point is located at:

$$
\gamma_{0}=\frac{1}{3}+\frac{2 Y_{1}}{9 \zeta}\left(\sqrt{3+\frac{Y_{1}^{2}}{\zeta^{2}}}+\frac{Y_{1}}{\zeta}\right) .
$$

This is exactly formula (34) of Ref. [11, with $Y_{1}=\psi \eta$.

From appendix $\mathrm{A}$ the expression of $\mathcal{R} e \mathbf{K}_{1}^{\prime}\left(\gamma_{0}\right)$ is given by:

$$
\begin{aligned}
\mathcal{R} e \mathbf{K}_{1}^{\prime}\left(\gamma_{0}\right) & =\zeta^{2}\left(1-\gamma_{0}\right)\left(\frac{Y_{1}}{\zeta \sqrt{\gamma_{0}}}-3+2 \frac{\sqrt{\gamma_{0}}}{\zeta} \omega_{01} \mathcal{R} e \frac{d Y_{1}}{d \omega}\right) \\
& =\zeta^{2}\left(1-\gamma_{0}\right)\left(\frac{Y_{1}}{\zeta \sqrt{\gamma_{0}}}-3+\theta \frac{3 \gamma_{0}+1}{2 \gamma_{0}} \frac{\mathcal{R} e Y_{1}^{\prime}}{\theta \mathcal{R} e Y_{1}^{\prime}-\mathcal{I} m Y_{1}^{\prime}}\right) .
\end{aligned}
$$

From the same appendix, the particular value of $Q_{1}\left(\gamma_{0}\right)$ when $D(\omega)=1$ is

$$
Q_{1}\left(\gamma_{0}\right)=\frac{B^{2}}{K_{2}\left(\gamma_{0}\right)}+C
$$

where

$$
\begin{aligned}
& B^{2}=\zeta^{4}\left(1-\gamma_{0}\right)^{2}\left(2+\frac{1-\gamma_{0}}{\gamma_{0}}+\frac{\left(1-\gamma_{0}\right)^{2}}{8 \gamma_{0}^{2}}\right) \\
& C=-\zeta^{2} \frac{1-\gamma_{0}}{4 \gamma_{0}}\left(3+\frac{3}{2} \frac{1-\gamma_{0}}{\gamma_{0}}\right) \\
& \mathbf{K}_{2}\left(\gamma_{0}\right)=2 \zeta \sqrt{\gamma_{0}}\left(1-\gamma_{0}\right)\left(Y_{2}-Y_{1}\right) .
\end{aligned}
$$

The quantities $B^{2}$ and $C$ are real and independent of frequency. Eq. (38) shows that when the quantity $\left(Y_{2}-Y_{1}\right)$ is modified, the sign of $\mathcal{R} e\left[Q_{1}\right]$ can change and the result of [9] is verified, as it can be seen by looking at Ref. [9] (see Eqs. (24) and (42)). Another expression of formula (38) can be found in appendix B, (Eq. (49)). 
After some calculations detailed in appendix B, the variation of frequency is given by the following formula:

$$
\frac{d \omega}{d \gamma}\left(\gamma_{0}\right)=\zeta \theta \frac{3 \gamma_{0}+1}{4 \gamma_{0} \sqrt{\gamma_{0}}\left(\theta \mathcal{R} e Y_{1}^{\prime}-\mathcal{I} m Y_{1}^{\prime}\right)}
$$

where

$$
\theta=-\frac{\mathcal{I} m Y_{2}}{\mathcal{R} e Y_{2}-Y_{1}+\frac{C}{B^{2}} 2 \zeta \sqrt{\gamma_{0}}\left(1-\gamma_{0}\right)\left|Y_{2}-Y_{1}\right|^{2}} .
$$

Let us remark that there is no frequency change if $Y_{2}$ is real. This is the case where no inharmonicity exists in the resonator. An example of such a resonator is the cylindrical bore of the clarinet when reed induced flow and dispersion are ignored.

We will now further investigate the previous formula (38), in the case where $Y_{2}$ is real. For this case, the sign of $\mathcal{R} e\left[Q_{1}\right]$ is given by (see appendix $\mathrm{B}$ ):

$$
\begin{aligned}
& \mathcal{R} e Q_{1}<0 \quad \text { if } \quad Y_{2}-Y_{1}<0 \quad \text { or } \quad Y_{2}-Y_{1}>\Gamma \text {, } \\
& \mathcal{R} e Q_{1}>0 \quad \text { if } \quad 0<Y_{2}-Y_{1}<\Gamma \text {, }
\end{aligned}
$$

where

$$
\Gamma=\frac{\zeta}{6 \sqrt{\gamma_{0}}} \frac{9 \gamma_{0}^{2}+6 \gamma_{0}+1}{\gamma_{0}+1} .
$$

Let us assume that $Y_{1}$ is small. This implies that $\gamma_{0}$ is close to $1 / 3$ from (18). For $\gamma_{0}=1 / 3, \Gamma=\zeta \sqrt{3} / 2$ and for $\gamma$ close to $\gamma_{0}$, Eq. (40) shows that the inequalities:

$$
Y_{1}+\mathcal{O}\left(\left|\gamma_{0}-\frac{1}{3}\right|\right)<Y_{2}<\frac{\sqrt{3} \zeta}{2}+Y_{1}-\mathcal{O}\left(\left|\gamma_{0}-\frac{1}{3}\right|\right)
$$

imply $\mathcal{R} e Q_{1}>0$. On the contrary, the inequalities

$$
Y_{2}<Y_{1}-\mathcal{O}\left(\left|\gamma_{0}-\frac{1}{3}\right|\right) \quad \text { or } \quad Y_{2}>\frac{\sqrt{3} \zeta}{2}+Y_{1}+\mathcal{O}\left(\left|\gamma_{0}-\frac{1}{3}\right|\right)
$$

imply $\mathcal{R} e Q_{1}<0$. Thus a bifurcation occurs when $Y_{1}$ and $Y_{2}$ are of the same order of magnitude and the nature of the bifurcation depends on the sign of $\mathcal{R} e\left[\mathbf{K}_{1}^{\prime}\left(\gamma_{0}\right)\right]$.

\section{Comparison with numerical approaches}

A numerical application has been done for the case of a model of tenor saxophone. The input impedance model of this conical instrument is described in details in appendix $\mathrm{C}$. The modulus of this impedance is plotted on figure 11. The reed dynamics is characterized by a second order low-pass filter as defined in 13] with a resonance frequency of $1500 \mathrm{~Hz}$ and a quality factor of 2.5. Here, the value of $\zeta$ is 0.3 .

\subsection{Frequency domain approach}

Let us introduce

$$
\omega_{1}^{\prime}=\frac{d \omega}{d \gamma}\left(\gamma_{0}\right)
$$

The values of $\gamma_{0}, \alpha, \omega_{1}^{0}, \omega_{1}^{\prime}$ of Eqs. (29) and (32) and the direction of bifurcation have been calculated using formulae (18) and the ones exposed in section 4 . Eq. (18) is satisfied by several values of $\omega$, giving different results for these quantities. Each solution corresponds to a register of the instrument, the first register being associated to the lowest frequency. The results for the first six registers are shown on Table 1. A comparison has been made with numerical results of harmonic balance, obtained using a recently developed approach [18]. From the computation, the values of $\gamma_{0}, \alpha, \omega_{1}^{0}, \omega_{1}^{\prime}$ have been extracted and are shown on Table 2. Results of these two methods are in excellent agreement. The small discrepancies observed are due to the numerical approach which must use a fit of the impedance into a truncated series of modes. This probably introduces a small error in the value of $Z(\omega)$ and hence on all the results. 

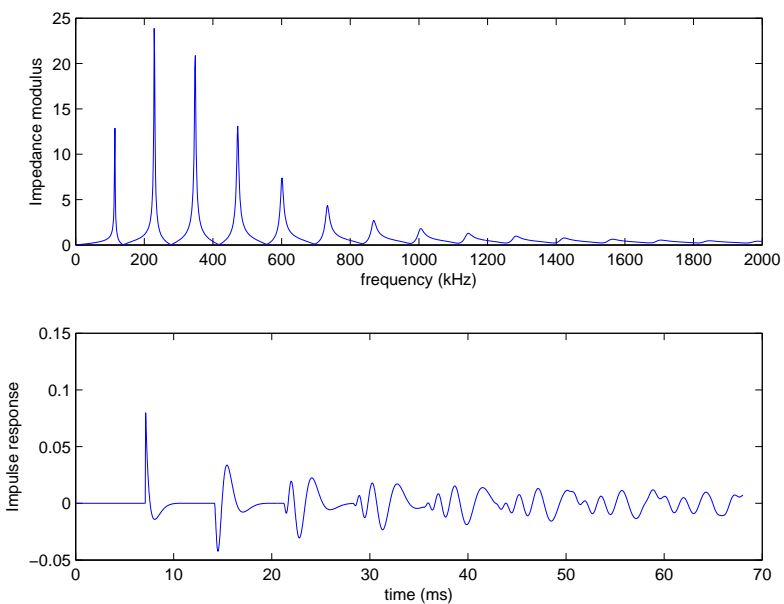

Figure 1: Top figure: modulus of the impedance $Z(\omega)$ of the saxophone used in the calculations of Sec. 6 , with respect to the frequency. It comes from the analytic expression (53). The first resonance peak is at around $114 \mathrm{~Hz}$. Bottom figure: impulse response associated (inverse Fourier transform of $Z(\omega)$ ).

\begin{tabular}{|c||c|c||c|c|c|}
\hline register & $\omega_{1}^{0}(\mathrm{~Hz})$ & $\omega_{1}^{\prime}(\mathrm{Hz})$ & $\gamma_{0}$ & $\sqrt{\alpha}$ & direction \\
\hline \hline 1 & 114.1058 & 21.6929 & 0.43923 & 0.38830 & + \\
\hline 2 & 228.9807 & 92.8306 & 0.38028 & 0.94362 & + \\
\hline 3 & 347.3668 & -5.9379 & 0.38504 & 0.90360 & + \\
\hline 4 & 471.0170 & -23.2183 & 0.41992 & 0.92096 & + \\
\hline 5 & 599.2781 & -18.4069 & 0.50481 & 0.98719 & + \\
\hline 6 & 730.6116 & -8.5105 & 0.69528 & 1.15041 & + \\
\hline
\end{tabular}

Table 1: Values for the parameters of Eqs. (29) and (32) associated with the tenor saxophone described in section 6 . they are given for the six first registers. The last column shows the direction of bifurcation, i.e. the sign of $\left(\gamma-\gamma_{0}\right)$.

\begin{tabular}{|c||c|c||c|c|c|}
\hline register & $\omega_{1}^{0}(\mathrm{~Hz})$ & $\omega_{1}^{\prime}(\mathrm{Hz})$ & $\gamma_{0}$ & $\sqrt{\alpha}$ & direction \\
\hline \hline 1 & 114.1083 & 21.865 & 0.43929 & 0.40222 & + \\
\hline 2 & 228.9834 & 93.22 & 0.38036 & 0.9225 & + \\
\hline 3 & 347.3710 & -6.01 & 0.38510 & 0.898 & + \\
\hline 4 & 471.0238 & -23.281 & 0.41989 & 0.89875 & + \\
\hline 5 & 599.2894 & -18.46 & 0.50453 & 0.921396 & + \\
\hline 6 & 730.6307 & -8.55 & 0.69408 & 1.058 & + \\
\hline
\end{tabular}

Table 2: Results computed using a numerical harmonic balance method presented in Ref. [18], giving values for the parameters of Eqs. (29) and (32) associated with the tenor saxophone described in section 6 . The discrepancy with results of table 1 increases with the value of the register but does not go higher than $0.003 \%$ for $\omega_{1}^{0}, 0.5 \%$ for $\omega_{1}^{\prime}, 0.2 \%$ for $\gamma_{0}$ and $10 \%$ for $\alpha$. 


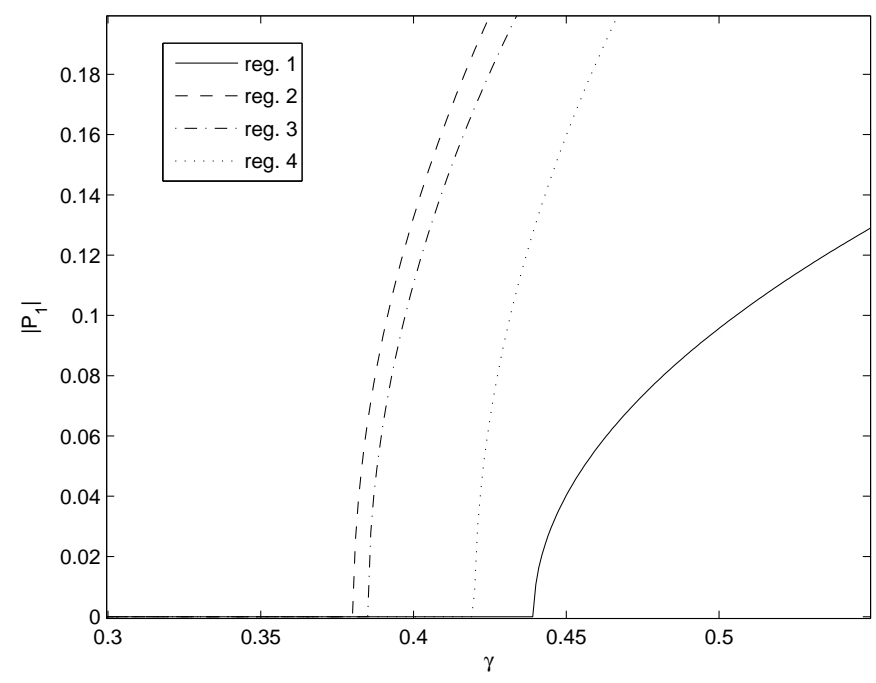

Figure 2: Amplitude of $P_{1}$ for the first four registers with respect to the blowing pressure, according to the values given in table 1. The accuracy of these curves is only guaranteed for a small interval around their respective oscillation threshold $\gamma_{0}$.

As shown in Fig. 2, an oscillation at the frequency of the second register can start for smaller values of $\gamma$ than the ones needed for the other registers. Between 0.3803 and 0.385 , it is the only small-oscillations solution (satisfying the Worman rule) possible for the system. Then, for larger values of the bifurcation parameter, supplementary solutions appear, associated to the third, fourth and first register, successively.

These solutions, satisfying relation (10), are shown to exist as they are confirmed by the numerical study, which is done without this assumption. This strengthens the justification of the Worman rule. Nevertheless, other solutions of the system, not satisfying this assumption, may exist and cannot be found by this analytical approach.

In this example, the appearance of the second regime before the other ones seems to be related to the fact that the highest peak of the input impedance is the second one. This kind of behaviour has been discussed for a more simplified case in Ref. [12]. But here, both the reed dynamics and the inharmonicity of the resonator imply that the oscillation frequency does not correspond to the maximum of the impedance peaks, as shown in the previous sections. Thus a counter example might well be found for this conjecture.

\subsection{Time domain simulation}

The synthesis algorithm used here is that presented by Guillemain et al. in Ref. [5] (see the equations in appendix (d). This scheme solves analytically the system of equations presented in the beginning of section 2, in the domain of sampled signals, by using a discrete time domain representation of the impedance. It is worth noting that the digital model, defined in the $z$-transform domain, possesses a continuous frequency representation.

The synthesis model has been used to run simulations where $\gamma$ linearly increases from 0 to a 0.384 and then remains constant. In order to get results close to the steady state regime situation, this evolution has been performed on a total duration of 380s. On Fig. 3, the top graph shows the value of blowing pressure $\gamma$ with respect to time. The bottom graph shows the amplitude of the first 4 harmonics. For this value of $\gamma$, an oscillation starts on the second register of the instrument after 200 seconds.

Let us notice that the frequency domain approach assumes a steady state where $\gamma$ is independent of time. This is not true for the time domain approach and even if oscillations are possible, they are not appearing exactly when $\gamma$ reaches the threshold. This is a consequence of the relatively fast variation of $\gamma$, giving no time for the oscillation to become established.

The value 0.384 is above the threshold of the second register but under the thresholds of the others registers given in the frequency domain approaches (see table 1 and 21). This hence confirms the results on the existence of an oscillation on the second register for this value of $\gamma$. 

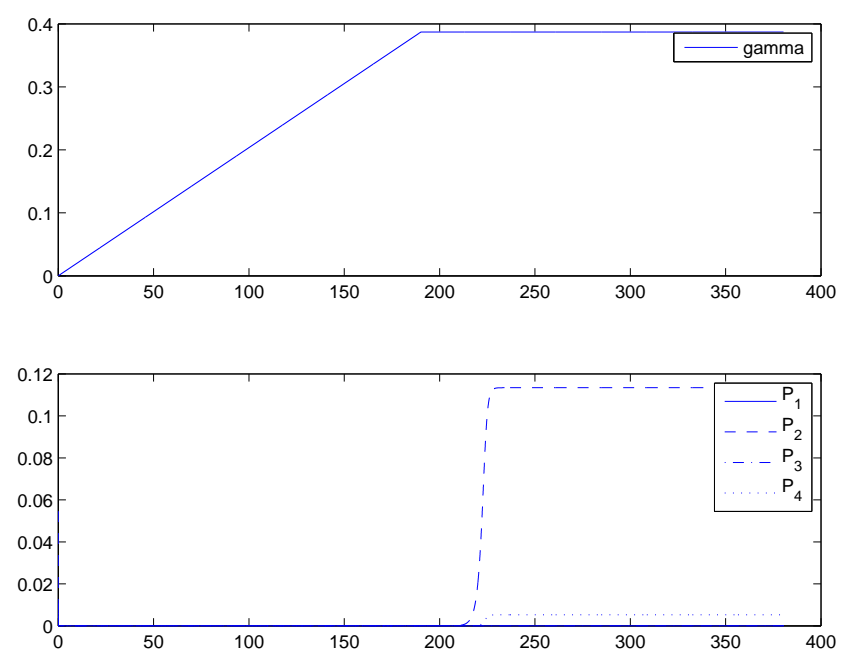

Figure 3: Top figure: value of the blowing pressure $\gamma$ with respect to time in seconds, increasing up to 0.384. Bottom figure: amplitude of the first 4 harmonics with respect to time, calculated using the time domain simulation.

General theory on the transition between static and oscillating regimes guarantees the existence of a (stable or unstable) oscillation around the first bifurcation appearing when $\gamma$ increases from zero. This is given by the Hopf bifurcation theorem (see e.g. Ref. [16, Chap. 1]). Before this bifurcation, the static regime is stable. Above this critical limit, it becomes unstable and the physical system usually encounters a change of regime toward the small amplitude oscillating solution (if this latter is stable). Nevertheless, when the parameter $\gamma$ increases further after this first bifurcation, nothing is said by the theory about the state of the system. Many solutions may appear from different phenomena which are not seen by the small oscillation analysis (for instance a period doubling of a solution) and the behaviour of the system can be represented by any one of these solutions, depending on the initial conditions. The present analytic approach, based on the Worman rule, gives several solutions for the system but not all of them while the time simulation is not limited to this. Thus there is no sense in comparing the results of both approaches for values of $\gamma$ higher than 0.385 .

\section{Conclusion}

This study has given several remarkable properties of woodwind instruments around the transition between stationary and oscillating regimes. Using the so called harmonic balance method, it is possible to take advantage of the polynomial nonlinear relationship to work directly and only in the frequency domain. Explicit calculations by this mean are possible and lead to analytical expressions of important characteristics of the bifurcation. The key point for the reduction of the system is to suppose a small oscillation regime and the Worman rule. Assuming so, several results of previous studies are confirmed by this more general approach and new relations are presented. An estimation of the error due to the small oscillation approximation is given for all the results.

It must be said that although many studies on wind instruments use it, the Worman rule is only proved for a truncated harmonic series. Nevertheless, it leads to coherent results in the present case of an infinite series: small oscillation regimes satisfying this rule are shown to exist via a comparison to numerical approaches.

This analytical approach gives a partial set of solutions of the system and by this mean is able to give an idea on the behaviour of reed instruments when physical parameters are tuned. Indeed, this method is satisfactory for the study of instruments which can be played with small oscillation, i.e. at pianissimo level. 


\section{Acknowledgments}

This work was supported by the French ANR research project CONSONNES.

\section{A Expression of the amplitude coefficient}

In this section, the quantities encountered in the publication are calculated to make explicit the dependence on the woodwind instruments parameters. At $\gamma=\gamma_{0}$, as defined in (8):

$$
\mathbf{H}_{2,1}=-\zeta^{2}\left(1-\gamma_{0}\right)\left(\frac{\left(1-\gamma_{0}\right)}{4 \gamma_{0}}+D_{1}\right)
$$

and

$$
\mathbf{H}_{1,2}+\mathbf{H}_{1,-1}=2 \zeta^{2} \gamma_{0} D_{-1} D_{2}-2 \zeta^{2}\left(1-\gamma_{0}\right)\left(D_{-1}+D_{2}\right)-2 Y_{-1} Y_{2} .
$$

One has also from (14) and since $\mathbf{K}_{-1}\left(\gamma_{0}\right)=0$ :

$$
\begin{aligned}
& Y_{2}=\frac{\mathbf{K}_{2}\left(\gamma_{0}\right)}{2 \zeta \sqrt{\gamma_{0}}\left(1-\gamma_{0}\right)}+\zeta \sqrt{\gamma_{0}}\left(D_{2}-\frac{\left(1-\gamma_{0}\right)}{2 \gamma_{0}}\right) \\
& Y_{-1}=\zeta \sqrt{\gamma_{0}}\left(D_{-1}-\frac{\left(1-\gamma_{0}\right)}{2 \gamma_{0}}\right)
\end{aligned}
$$

and

$$
Y_{2} Y_{-1}=\frac{\mathbf{K}_{2}\left(\gamma_{0}\right)}{2\left(1-\gamma_{0}\right)}\left(D_{-1}-\frac{1-\gamma_{0}}{2 \gamma_{0}}\right)+\zeta^{2} \gamma_{0}\left(D_{2} D_{-1}-\frac{1-\gamma_{0}}{2 \gamma_{0}}\left(D_{2}+D_{-1}\right)+\left(\frac{1-\gamma_{0}}{2 \gamma_{0}}\right)^{2}\right) .
$$

Then

$$
\mathbf{H}_{1,2}+\mathbf{H}_{1,-1}=\frac{-\mathbf{K}_{2}\left(\gamma_{0}\right)}{\left(1-\gamma_{0}\right)}\left(D_{-1}-\frac{1-\gamma_{0}}{2 \gamma_{0}}\right)-\zeta^{2}\left(1-\gamma_{0}\right)\left(D_{-1}+D_{2}+\frac{1-\gamma_{0}}{2 \gamma_{0}}\right)
$$

and

$$
\begin{aligned}
\left(\mathbf{H}_{1,2}+\mathbf{H}_{1,-1}\right) \mathbf{H}_{2,1}= & \mathbf{K}_{2}\left(\gamma_{0}\right) \zeta^{2}\left(\left|D_{1}\right|^{2}+\frac{1-\gamma_{0}}{4 \gamma_{0}}\left(D_{-1}-2 D_{1}\right)-\frac{\left(1-\gamma_{0}\right)^{2}}{8 \gamma_{0}^{2}}\right)+ \\
& +\zeta^{4}\left(1-\gamma_{0}\right)^{2}\left(\left|D_{1}\right|^{2}+D_{1} D_{2}+\left(3 D_{1}+D_{2}\right) \frac{1-\gamma_{0}}{4 \gamma_{0}}+\frac{\left(1-\gamma_{0}\right)^{2}}{8 \gamma_{0}^{2}}\right) .
\end{aligned}
$$

One has also:

$$
\begin{aligned}
& \mathbf{H}_{0,1}+\mathbf{H}_{0,-1}=2 \zeta^{2}\left[\gamma_{0}\left|D_{1}\right|^{2}-\left(1-\gamma_{0}\right)\left(D_{1}+\overline{D_{1}}\right)\right]-2\left|Y_{1}\right|^{2}=-\zeta^{2}\left(1-\gamma_{0}\right)\left(D_{1}+\overline{D_{1}}+\frac{1-\gamma_{0}}{2 \gamma_{0}}\right), \\
& \frac{\mathbf{H}_{0,1}+\mathbf{H}_{0,-1}}{\sqrt{u_{00}}} Y_{1}=-\frac{\zeta Y_{1}}{\sqrt{\gamma_{0}}}\left(D_{1}+\overline{D_{1}}+\frac{1-\gamma_{0}}{2 \gamma_{0}}\right),
\end{aligned}
$$

which can be written:

$$
\begin{aligned}
\frac{\mathbf{H}_{0,1}+\mathbf{H}_{0,-1}}{\sqrt{u_{00}}} Y_{1} & =-\zeta^{2}\left(D_{1}-\frac{1-\gamma_{0}}{2 \gamma_{0}}\right)\left(D_{1}+\overline{D_{1}}+\frac{1-\gamma_{0}}{2 \gamma_{0}}\right) \\
& =-\zeta^{2}\left(D_{1}^{2}+\left|D_{1}\right|^{2}-\frac{1-\gamma_{0}}{2 \gamma_{0}}\left(D_{-1}+\frac{1-\gamma_{0}}{2 \gamma_{0}}\right)\right) .
\end{aligned}
$$

From (6)

$$
C_{1,0,1}+C_{1,0,-1}+C_{1,2,1}=-\zeta^{2}\left(2\left|D_{1}\right|^{2}+\left(D_{1}\right)^{2}\right) .
$$

Since $\mathbf{K}_{1}\left(\gamma_{0}\right)=0$ it can be written

$$
\begin{aligned}
\mathbf{K}_{2}\left(\gamma_{0}\right) & =\mathbf{K}_{2}\left(\gamma_{0}\right)-\mathbf{K}_{1}\left(\gamma_{0}\right)=2 \zeta \sqrt{\gamma_{0}}\left(1-\gamma_{0}\right)\left(Y_{2}-Y_{1}\right)-2 \zeta^{2} \gamma_{0}\left(1-\gamma_{0}\right)\left(D_{2}-D_{1}\right) \\
& =2 \zeta \sqrt{\gamma_{0}}\left(1-\gamma_{0}\right)\left[\left(Y_{2}-Y_{1}\right)-\zeta \sqrt{\gamma_{0}}\left(D_{2}-D_{1}\right)\right] .
\end{aligned}
$$


Let us introduce

$$
\begin{aligned}
B^{2}= & \zeta^{4}\left(1-\gamma_{0}\right)^{2}\left(\left|D_{1}\right|^{2}+D_{1} D_{2}+\left(3 D_{1}+D_{2}\right) \frac{1-\gamma_{0}}{4 \gamma_{0}}+\frac{\left(1-\gamma_{0}\right)^{2}}{8 \gamma_{0}^{2}}\right) \\
C= & -\zeta^{2}\left(2\left|D_{1}\right|^{2}+D_{1}^{2}\right)+\zeta^{2}\left(D_{1}^{2}+\left|D_{1}\right|^{2}-\frac{1-\gamma_{0}}{2 \gamma_{0}}\left(D_{-1}+\frac{1-\gamma_{0}}{2 \gamma_{0}}\right)\right) \\
& +\zeta^{2}\left(\left|D_{1}\right|^{2}+\frac{1-\gamma_{0}}{4 \gamma_{0}}\left(D_{-1}-2 D_{1}\right)-\frac{\left(1-\gamma_{0}\right)^{2}}{8 \gamma_{0}^{2}}\right) \\
= & -\zeta^{2} \frac{1-\gamma_{0}}{4 \gamma_{0}}\left(2 D_{1}+\overline{D_{1}}+\frac{3}{2} \frac{1-\gamma_{0}}{\gamma_{0}}\right),
\end{aligned}
$$

then Eq. (20) becomes

$$
Q_{1}=\frac{B^{2}}{\mathbf{K}_{2}\left(\gamma_{0}\right)}+C \text { and } \mathcal{R} e Q_{1}=\frac{\mathcal{R} e \mathbf{K}_{2}\left(\gamma_{0}\right) \mathcal{R} e B^{2}+\mathcal{I} m \mathbf{K}_{2}\left(\gamma_{0}\right) \mathcal{I} m B^{2}}{\left|\mathbf{K}_{2}\left(\gamma_{0}\right)\right|^{2}}+\mathcal{R} e C .
$$

Eventually let us write the expression:

$$
\begin{aligned}
\mathbf{K}_{1}^{\prime}\left(\gamma_{0}\right) & =\zeta^{2}\left(1-\gamma_{0}\right)\left(\frac{Y_{1}}{\zeta \sqrt{\gamma_{0}}}-1-2 D_{1}+2 \frac{\omega_{1}^{\prime} \sqrt{\gamma_{0}}}{\zeta} \frac{d Y_{1}}{d \omega}-2 \gamma_{0} \omega_{1}^{\prime} \frac{d D_{1}}{d \omega}\right) \\
& =\zeta^{2}\left(1-\gamma_{0}\right)\left(-\frac{1}{2 \gamma_{0}}-\frac{1}{2}-D_{1}+2 \frac{\omega_{1}^{\prime} \sqrt{\gamma_{0}}}{\zeta} \frac{d Y_{1}}{d \omega}\left(\gamma_{0}\right)-2 \gamma_{0} \omega_{1}^{\prime} \frac{d D_{1}}{d \omega}\left(\gamma_{0}\right)\right)
\end{aligned}
$$

where $\omega_{1}^{\prime}$ is the derivative of $\omega$ with respect to $\gamma$ at $\gamma_{0}$.

\section{B Calculations with no reed dynamics}

Let us start from Eq. (38) with the assumption $D(\omega)=1$ for all $\omega$. Only $\mathbf{K}_{2}\left(\gamma_{0}\right)$ may be a complex number because $Y_{2}$ is a complex quantity, therefore

$$
\begin{aligned}
\theta & =-\frac{\mathcal{I} m\left[\mathbf{K}_{2}\left(\gamma_{0}\right)\right]}{\mathcal{R} e\left[\mathbf{K}_{2}\left(\gamma_{0}\right)\right]+C\left|\mathbf{K}_{2}\left(\gamma_{0}\right)\right|^{2} / B^{2}}=-\frac{\mathcal{I} m\left[Y_{2}\right]}{\mathcal{R} e\left[Y_{2}\right]-Y_{1}+\frac{C\left|\mathbf{K}_{2}\left(\gamma_{0}\right)\right|^{2}}{2 \zeta \sqrt{\gamma_{0}}\left(1-\gamma_{0}\right) B^{2}}} \\
& =-\frac{\mathcal{I} m\left[Y_{2}\right]}{\mathcal{R} e\left[Y_{2}\right]-Y_{1}+\frac{C}{B^{2}} 2 \zeta \sqrt{\gamma_{0}}\left(1-\gamma_{0}\right)\left|Y_{2}-Y_{1}\right|^{2}} .
\end{aligned}
$$

Eq. (34) becomes:

$$
\begin{aligned}
& \frac{\partial \mathbf{K}_{1}}{\partial \gamma}\left(\omega\left(\gamma_{0}\right), \gamma_{0}\right)=-\zeta^{2} \frac{\left(1-\gamma_{0}\right)}{2 \gamma_{0}}\left(3 \gamma_{0}+1\right), \\
& \frac{\partial \mathbf{K}_{1}}{\partial \omega}\left(\omega\left(\gamma_{0}\right), \gamma_{0}\right)=2 \zeta\left(1-\gamma_{0}\right) Y_{1}^{\prime} \sqrt{\gamma_{0}}
\end{aligned}
$$

which leads to:

$$
\frac{d \omega}{d \gamma}\left(\gamma_{0}\right)=\zeta \theta \frac{3 \gamma_{0}+1}{4 \gamma_{0} \sqrt{\gamma_{0}}\left(\theta \mathcal{R} e Y_{1}^{\prime}-\mathcal{I} m Y_{1}^{\prime}\right)}
$$

Let us look at the direction of bifurcation. Here since $B^{2}$ and $C$ are real,

$$
\mathcal{R} e Q_{1}=\frac{\mathcal{R} e \mathbf{K}_{2}\left(\gamma_{0}\right) B^{2}}{\left|\mathbf{K}_{2}\left(\gamma_{0}\right)\right|^{2}}+C
$$

with

$$
\left|\mathbf{K}_{2}\left(\gamma_{0}\right)\right|^{2}=4 \zeta^{2} \gamma_{0}\left(1-\gamma_{0}\right)^{2}\left|Y_{2}-Y_{1}\right|^{2}
$$

so

$$
\mathcal{R} e Q_{1}=\zeta^{2} \frac{1-\gamma_{0}}{2}\left[\frac{\zeta}{\sqrt{\gamma_{0}}}\left(2+\frac{1-\gamma_{0}}{\gamma_{0}}+\frac{\left(1-\gamma_{0}\right)^{2}}{8 \gamma_{0}^{2}}\right) \frac{\mathcal{R} e Y_{2}-Y_{1}}{\left|Y_{2}-Y_{1}\right|^{2}}-\frac{1}{2 \gamma_{0}}\left(3+\frac{3}{2} \frac{1-\gamma_{0}}{\gamma_{0}}\right)\right]
$$


Let us investigate the $\operatorname{sign}$ of $\mathcal{R} e Q_{1}$ and solve the equation $\mathcal{R} e Q_{1}=0$; This gives the condition:

$$
\begin{aligned}
\frac{\mathcal{R} e Y_{2}-Y_{1}}{\left|Y_{2}-Y_{1}\right|^{2}} & =\frac{1}{2 \zeta \sqrt{\gamma_{0}}} \frac{3+\frac{3}{2} \frac{1-\gamma_{0}}{2+\frac{1-\gamma_{0}}{\gamma_{0}}+\frac{\left(1-\gamma_{0}\right)^{2}}{8 \gamma_{0}^{2}}}}{} \\
& =\frac{6 \sqrt{\gamma_{0}}}{\zeta} \frac{\gamma_{0}+1}{9 \gamma_{0}^{2}+6 \gamma_{0}+1} .
\end{aligned}
$$

If the notation $y_{r}=\mathcal{R} e\left(Y_{2}-Y_{1}\right), y_{i}=\mathcal{I} m\left(Y_{2}-Y_{1}\right)$ is introduced and

$$
\Gamma=\frac{\zeta}{6 \sqrt{\gamma_{0}}} \frac{9 \gamma_{0}^{2}+6 \gamma_{0}+1}{\gamma_{0}+1}
$$

the previous relationship becomes:

$$
y_{r}^{2}-\Gamma y_{r}+y_{i}^{2}=0 \Leftrightarrow y_{r}=\frac{\Gamma}{2} \pm \frac{\sqrt{\Gamma^{2}+4 y_{i}^{2}}}{2} .
$$

As a consequence

$$
\begin{array}{rrr}
\mathcal{R} e Q_{1}<0 \quad \text { if } & y_{r}<\frac{\Gamma}{2}-\frac{\sqrt{\Gamma^{2}+4 y_{i}^{2}}}{2} \text { or } \quad y_{r}>\frac{\Gamma}{2}+\frac{\sqrt{\Gamma^{2}+4 y_{i}^{2}}}{2}, \\
\mathcal{R} e Q_{1}>0 \text { if } & \frac{\Gamma}{2}-\frac{\sqrt{\Gamma^{2}+4 y_{i}^{2}}}{2}<y_{r}<\frac{\Gamma}{2}+\frac{\sqrt{\Gamma^{2}+4 y_{i}^{2}}}{2} .
\end{array}
$$

\section{Input impedance of the model of saxophone}

The impedance model of the conical resonator considered in section 6 corresponds to that discussed e.g by Dalmont 19]:

$$
Z(\omega)=\frac{1}{\frac{1}{j \tan (k L)}+\frac{c}{j \omega x_{e}}+\frac{j \omega x_{e}}{3 c}}
$$

where $L=1.2 \mathrm{~m}$ is the length of the truncated cone, $c=340 \mathrm{~m} / \mathrm{s}$ the speed of sound and $k$ is the wavenumber. The volume of the mouthpiece is assumed to be equal to this of the missing part of the cone, of length $x_{e}=0.26573 \mathrm{~m}$. Let us introduce $F_{e}=44100 \mathrm{~Hz}$, the sampling frequency for the numerical model. In order to use a digital model taking dissipation into account, the term $R$, equivalent to a reflection coefficient, is introduced as:

$$
j \tan (k L)=\frac{1+R}{1-R}
$$

and is approximated by

$$
R=\frac{-b_{0} \exp \left(-j \omega D / F_{e}\right)}{1-a_{1} \exp (-j \omega / F e)} .
$$

The quantities $b_{0}=0.20336$ and $a_{1}=0.78671$ are related to the viscothermal and radiation losses, and $D$ is a delay related to the length of the tube $(D=126$ samples). Eventually, the second and third term of the denominator of (53) are estimated by the bilinear transform, $j \omega$ being replaced by:

$$
j \omega=2 F_{e} \frac{1-\exp (-j \omega / F e)}{1+\exp (-j \omega / F e)}
$$

As noticed in Ref. [20] (see p. 268), if the impedance model does not satisfy the hypothesis $Z(0)=0$, the parameter $\gamma$ can be redefined, as the difference between the mouth pressure and the mean pressure inside the mouthpiece, (instead of the mouth pressure). Then the associated impedance $\widetilde{Z}$ satisfies $\widetilde{Z}(0)=0$. 


\section{References}

[1] Worman, W.E., Self-sustained nonlinear oscillations of medium amplitude in clarinet-like systems, PhD thesis, 1971, Case Western Reserve University.

[2] R. T. Schumacher, Self-sustained oscillations of the clarinet: an integral equation approach, Acustica 40, 298-309 (1978).

[3] J. Gilbert, J. Kergomard, E. Ngoya, Calculation of the steady-state oscillations of a clarinet using the harmonic balance technique, J. Acoust. Soc. Am. 86 (1), 35-41, (1989).

[4] Schumacher, R. T., Ab initio calculations of the oscillation of a clarinet (1981), Acustica, 48(71-85).

[5] Ph. Guillemain, J. Kergomard, T. Voinier, "Real-time synthesis of clarinet-like instruments using digital impedance models", J. Acout. Soc. Am., 118(1), 483-494, (2005).

[6] Debut V., Deux études d'un instrument de musique de type clarinette: analyse des fréquences propres du résonateur et calcul des auto-oscillations par décomposition modale. $\mathrm{PhD}$ thesis, Université de la Méditerranée, 2004.

[7] C. Fritz, S. Farner, J. Kergomard, Some aspects of the harmonic balance method applied to the clarinet, Applied Acoustics, 65 (2004), pp. 1155-1180.

[8] S. Farner, C. Vergez, J. Kergomard, A. Lizée, Contribution to harmonic balance calculations of selfsustained periodic oscillations with focus on single-reed instruments, J. Acoust. Soc. Am. 119 (3), pp. 1794-1804 (2006).

[9] N. Grand, J. Gilbert, F. Laloë, Oscillation threshold of woodwind instruments, Acustica - Acta Acustica, 82 (1996), 137-151.

[10] A. I. Mees, Describing Functions: Ten Years On, IMA J. App. Math. (1984) 32,221-223.

[11] Kergomard, J. and Ollivier, S. and Gilbert, J., Calculation of the Spectrum of Self-Sustained Oscillators Using a Variable Truncation Method: Application to Cylindrical Reed Instruments, Acta Acustica, 86, 685-703 (2000).

[12] Dalmont J.-P., Gilbert J., Kergomard J., Reed Instruments, from Small to Large Amplitude Periodic Oscillations and the Helmholtz Motion Analogy, Acustica - Acta acustica, 86,671-684, 2000.

[13] Silva F., Kergomard J., Vergez C., Gilbert J., Interaction of reed and acoustic resonator in clarinetlike systems, J. Acoust. Soc. Am.(2008).

[14] Fletcher N.H., Rossing T.D., The physics of Musical Instruments, Springer, (1991).

[15] Kergomard J., Chaigne A., Acoustique des instruments de musique, Belin, 2008.

[16] B. D. Hassard, N. D. Kazarinoff, Y-H. Wan, Theory and Applications of Hopf Bifurcation, London Mathematical Society Lecture Note Series, 41, Cambridge University Press, 1981.

[17] Kergomard J., Gilbert J., Analyse de quelques aspects du rôle de l'anche d'un instrument à vent cylindrique, Proceedings of the 5th French congress on acoustics, Lausanne, pp. 294-297 (2000).

[18] B. Cochelin, C. Vergez, A high order purely frequency-based harmonic balance formulation for continuation of periodic solutions, J. Sound Vib., under revision.

[19] J.-P. Dalmont, B. Gazengel, J. Gilbert, J. Kergomard, "Some aspects of tuning and clean intonation in woodwinds", Applied Acoustics 46, 19-60 (1995).

[20] A. Hirschberg, J. Kergomard, G. Weinreich, Mechanics of musical instruments, CISM courses and lectures 335, Springler-Verlag, 1995. 